\title{
APPLICATION OF MACHINE LEARNING TO IMPROVE THE PERFORMANCE OF A PRESSURE-CONTROLLED SYSTEM
}

\author{
Fabian Kreutmayr*, Markus Imlauer \\ HAWE Hydraulik SE, Einsteinring 17, 85609 Aschheim / München \\ * Corresponding author: Tel.: +49 (89) 379100 - 1149; E-mail address: f.kreutmayr@hawe.de
}

\begin{abstract}
Due to the robustness and flexibility of hydraulic components, hydraulic control systems are used in a wide range of applications under various environmental conditions. However, the coverage of this broad field of applications often comes with a loss of performance. Especially when conditions and working points change often, hydraulic control systems cannot work at their optimum. Flexible electronic controllers in combination with techniques from the field of machine learning have the potential to overcome these issues. By applying a reinforcement learning algorithm, this paper examines whether learned controllers can compete with an expert-tuned solution. Thereby, the method is thoroughly validated by using simulations and experiments as well.
\end{abstract}

Keywords: Hydraulic control systems, Machine learning, Reinforcement learning

\section{INTRODUCTION}

Since hydraulic control systems are used in various fields with a wide range of different operating conditions, e.g. range of oil temperature, range of pressure, and type of fluid, the design of these systems is a challenging procedure. Hereby, the availability of hydraulic components that can deal with this variety of requirements is crucial. For this purpose, a major design criterion for hydraulic components is that they remain flexible. This flexibility is conventionally achieved at the expense of performance, which influences the whole hydraulic control system.

Moreover, fixed inflexible mechanic controllers are still used for many applications. Whilst these are self-sufficient and robust against influences like temperature changes or heavy vibrations, adaptability to different working points is not given. Additionally, these controllers suffer from abrasion.

By using electronic instead of mechanic controllers, these drawbacks can be compensated, because their parameters can be changed easily. Though, since the system dynamics are often partially or completely unknown, tuning these control parameters is time consuming and may result in non-optimal solutions. So, the application of methods from the field of artificial intelligence, i.e. machine learning, can help to reduce the effort of complex parameter tuning. Especially reinforcement learning (RL) algorithms have already shown promising results for solving various control tasks $[1,2,3]$.

This work examines the applicability of such RL algorithms to control hydraulic systems. For this purpose, a mathematical model of an example system is derived and implemented in simulation. Afterwards, the deep deterministic policy gradient (DDPG) [4] algorithm is applied to tune the parameters of a PID controller.

In order to validate this method, simulations and measurements are done. By performing the same control task on a test rig, the method's applicability to a real hydraulic system is evaluated.

\section{SYSTEM}

In the following, the system that is used to evaluate the previously introduced approach is described.

\subsection{Description}

The control system used in this work contains of a hydro motor surrounded by two proportional valves. The first valve is on its a-side connected to a pump that delivers a constant pressure. On its $\mathrm{b}$-side, the valve is connected to the a-side of the 
hydro motor. On the b-side of the motor follows the a-side of the second valve, while the b-side of this valve is connected to a tank. A representation of this system can be seen in Figure 1.

The goal of the control task is to achieve a certain pressure $p_{V, 1, b} \in \mathbb{R}_{+}$between the first valve and the motor. Hereby, the first valve controls the volumetric flow rate via a predefined lookup-table. The second valve is expected to control the pressure $p_{V, 1, b}$. Moreover, the hydro motor simulates unknown disturbances that may influence the system performance.

\subsection{Mathematical Model}

In this chapter, a mathematical description of the proposed system is introduced. Hereby, formulas for each component are used to derive a complete state-space model of the system.

\section{Hydraulic motor}

The hydraulic motor can be described by the following differential equation:

$\ddot{\varphi}=\frac{1}{I}\left(\frac{V_{d}}{2 \pi}\left(p_{a}-p_{b}\right)-d \dot{\varphi}-M\right)$.

With $\dot{\varphi} \in \mathbb{R}$ as angular velocity, $\ddot{\varphi} \in \mathbb{R}$ as angular acceleration, $I \in \mathbb{R}$ as the moment of inertia, $V_{d} \in \mathbb{R}$ as reference volume, $p_{a}, p_{b} \in \mathbb{R}_{+}$ as the pressure on a- and b-side, $d \in \mathbb{R}$ as the damping factor, and $M \in \mathbb{R}$ as an external torque. Moreover, the volumetric flow rate $Q_{M} \in \mathbb{R}$ inside the motor is defined as

$Q_{M}=\dot{\varphi} \frac{V_{d}}{2 \pi}$.

\section{Proportional valve}

Since both proportional valves are of the same type, also their dynamics are similar. Thus, they can be described as follows:
$Q_{V, i}=\alpha A_{i}\left(I_{V, i}\right) \sqrt{2 \frac{\Delta p_{V, i}}{\rho}}$.

Here, $Q_{V, i} \in \mathbb{R}$ is the volumetric flow through the valve, $\alpha \in \mathbb{R}$ is the discharge coefficient, $A_{i}\left(I_{V, i}\right) \in \mathbb{R}_{+}$is the valve opening, $I_{V, i} \in \mathbb{R}$ is the control current that is applied on the valve, $\Delta p_{V, i} \in \mathbb{R}_{+}$is the difference between the pressures $p_{V, i, a} \in \mathbb{R}_{+}$and $p_{V, i, b} \in \mathbb{R}_{+}$, and $\rho \in$ $\mathbb{R}_{+}$is the fluid density. Furthermore, the index $i=\{1,2\}$ refers to one of the two valves.

As written above, the opening of the proportional valve depends on the control current $I_{V, i}$ that is applied. However, the correlation between $A_{i}$ and $I_{V, i}$ is nonlinear. Additionally, the derivation of a mathematical connection is difficult, since different effects, e.g. magnetization hysteresis, friction, and spring properties, need to be considered.

Thus, $A_{i}\left(I_{V, i}\right)$ is approximated by the hysteresis in Figure 2. In the simulation, those values are stored in a look-up table. While this might not cover the detailed physical behavior of the valve, experiments have shown that this simplification is sufficient for the purpose of this paper.

\section{Hydraulic pipes}

Another significant influence in the system comes from the hydraulic pipes that connect the motor and the valves. Moreover, the pressure sensors, which are necessary to achieve the control objective, are located in these pipes. As proposed in literature [5], the rate of change of the pressure $p_{V, 1, b}$ is defined as

$\dot{p}_{V, 1, b}=\frac{1}{c_{1}}\left(Q_{V, 1}-Q_{M}\right)$.

With $c_{1} \in \mathbb{R}_{+}$as constant for the combination of bulk modulus and fluid volume. Analogously, the rate of change of $p_{\mathrm{V}, 2, \mathrm{a}}$ can be written as

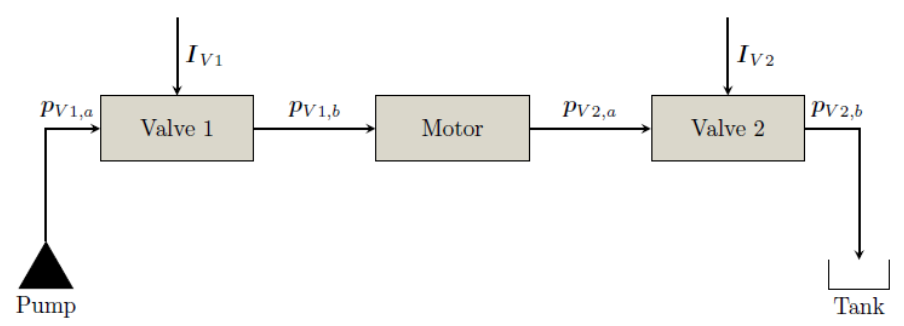

Figure 1: Simplified visualization of the hydraulic system that is used in this work. 
$\dot{p}_{V, 2, a}=\frac{1}{c_{2}}\left(Q_{M}-Q_{V, 2}\right)$.

Hence, both pressures $p_{V, 1, b}$ and $p_{V, 2, a}$ can be calculated from the integral of Equation 4 and 5, respectively. This results in

$p_{V, 1, b}=\frac{1}{c_{1}} \int\left(Q_{V, 1}-Q_{M}\right) d t$

for $p_{V, 1, b}$ and

$p_{V, 2, a}=\frac{1}{c_{2}} \int\left(Q_{M}-Q_{V, 2}\right) d t$

for $p_{V, 2, a}$.

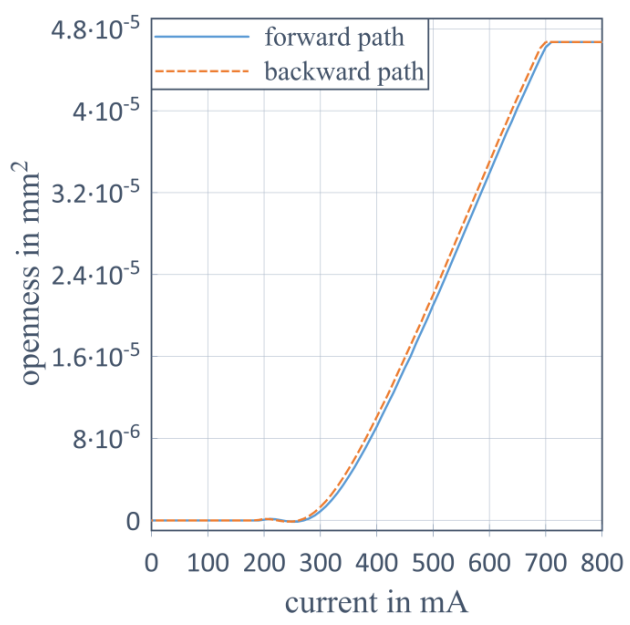

Figure 2: Hysteresis of the valve opening A. The width of the hysteresis is $10 \mathrm{~mA}$.

\subsection{State-Space Model}

As already mentioned in the previous section, the presented formulas are used for a state-space representation. Because of the nonlinear and time-variant behavior of the system, e.g. through the hysteresis caused by the proportional magnet, the system is formulated in the form of a nonlinear dynamical system, which is:

$\dot{x}=f(x(t), u(t))$.

Here, $\dot{x}$ is the derivative of the state vector $x$, which is defined as follows:

$x=\left[\begin{array}{c}\varphi \\ \dot{\varphi} \\ p_{V, 1, b} \\ p_{V, 2, a}\end{array}\right]$.

Thus, $\dot{x}$ is defined as $\dot{x}=\left[\begin{array}{c}\dot{\varphi} \\ \ddot{\varphi} \\ \dot{p}_{V, 1, b} \\ \dot{p}_{V, 2, a}\end{array}\right]$.

In order to achieve a concise state-space description, it is necessary to transform the formulas from the previous section into a consistent form. Since $p_{a}=p_{V, 1, b}$ and $p_{b}=$ $p_{V, 2, a}$, Equation 1 can be reformulated as

$\ddot{\varphi}=\frac{1}{I}\left(\frac{V_{d}}{2 \pi}\left(p_{V, 1, b}-p_{V, 2, a}\right)-d \dot{\varphi}-M\right)$.

Moreover, Equation 2 and Equation 3 can be inserted in Equation 4, which results in

$\dot{p}_{V, 1, b}=\frac{1}{c_{1}}\left(\alpha A_{1}\left(I_{V, 1}\right) \sqrt{2 \frac{\Delta p_{V, 1}}{\rho}}-\dot{\varphi} \frac{V_{d}}{2 \pi}\right)$.

Analogously, Equation 2 and Equation 3 can be inserted in Equation 5, which leads to

$\dot{p}_{V, 2, a}=\frac{1}{c_{2}}\left(\dot{\varphi} \frac{V_{d}}{2 \pi}-\alpha A_{2}\left(I_{V, 2}\right) \sqrt{2 \frac{\Delta p_{V, 2}}{\rho}}\right)$.

Finally, the state-space description of the valvemotor-valve system is:

$\dot{x}=\left[\begin{array}{c}\dot{\varphi} \\ \frac{1}{I}\left(\frac{V_{d}}{2 \pi}\left(p_{V, 1, b}-p_{V, 2, a}\right)-d \dot{\varphi}-M\right) \\ \frac{1}{c_{1}}\left(\alpha A_{1}\left(I_{V, 1}\right) \sqrt{2 \frac{\Delta p_{V, 1}}{\rho}}-\dot{\varphi} \frac{V_{d}}{2 \pi}\right) \\ \frac{1}{c_{2}}\left(\dot{\varphi} \frac{V_{d}}{2 \pi}-\alpha A_{2}\left(I_{V, 2}\right) \sqrt{2 \frac{\Delta p_{V, 2}}{\rho}}\right)\end{array}\right]$.

\subsection{Controller}

As described in the previous sections, the valve opening is controlled by the electric current $I_{V, i}$.

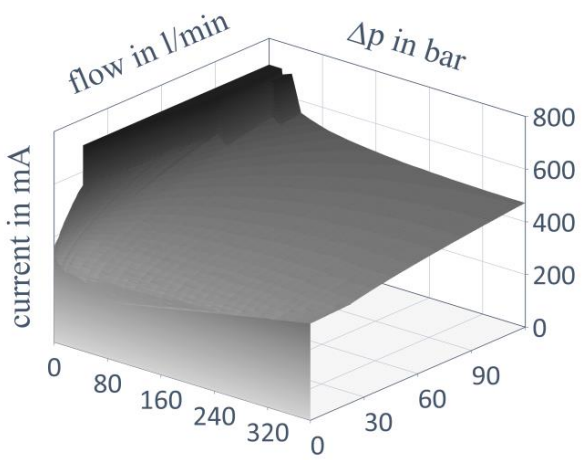

Figure 3: 3D-plot of the look-up table for the openloop control of valve 1 . 


\section{Valve 1: Open-loop control}

The first valve is controlled by using a static lookup table. Hereby, the pressure difference $\Delta p_{V, 1}$ and the desired flow $Q$ is used as an input for the table. The combination of these values results in an electric current $I_{V, 1}$, which leads to an opening $A_{1}$ that leads to the desired flow. Since this is a simple open-loop control approach, there is no guarantee that the resulting flow corresponds with the desired flow.

Though, since the look-up table was created experimentally and, thus, is fitted to the used valve type, it shows a reasonable performance. A visualization of the look-up table used for openloop control is shown in Figure 3.

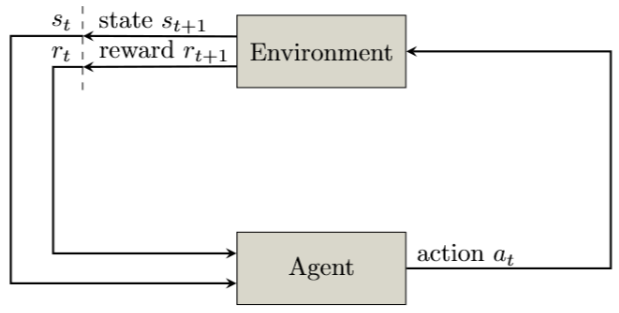

Figure 4: Basic reinforcement learning structure.

\section{Valve 2: Closed-loop control}

For the second valve, which controls the pressure $p_{V, 1, b}$, a closed-loop control strategy is used. Since the PID controller is a standard in industrial applications and in hydraulics, it is also used in this work. Moreover, the usage of a state-of-theart control structure gives a reasonable benchmark for the evaluation of the approach introduced in this paper.

While there are various forms of the PID controller available, this work uses the standard form, given by the following mathematical expression:

$u=K_{p} e+K_{i} \int e d \tau+K_{d} \dot{e}$

Here, $u \in R$ is the controller output, $K_{p} \in \mathbb{R}$ is the proportional gain, $K_{i} \in \mathbb{R}$ is the integral gain, $K_{d} \in \mathbb{R}$ is the differential gain, $e \in \mathbb{R}$ is the control error, and $\dot{e} \in \mathbb{R}$ is the derivative of the control error. Moreover, the control error is defined as:

$e=p_{V, 1, b, r e f}-p_{V, 1, b}$

with $p_{V, 1, b, r e f} \in \mathbb{R}_{+}$as the reference signal.

\section{DEEP DETERMINISTIC GRADIENT}

This section provides both a short introduction into the basics of reinforcement learning [6] and the information about the deep deterministic policy gradient algorithm, which is used in this work.

\subsection{Reinforcement learning}

The aim of reinforcement learning (RL) is that an agent learns through interaction with an environment (see Figure 4). This environment can be modeled as a Markov decision process (MDP), which is defined as a quadruple $(S, A, f, J)$. Thereby, $S \in \mathbb{R}^{\mathrm{n}}$ is the state space vector, $A \in \mathbb{R}^{m}$ is the action space vector, $f: S \times A \rightarrow S$ is the transition function, and $f: S \times A \rightarrow \mathbb{R}$ is the reward function. Furthermore, $n, m \in \mathbb{N}$ denote as the number of states and actions, respectively.

In each timestep $t$, the agent receives information about the state $s_{t} \in S$ from the environment and the agent chooses an appropriate action $a_{t} \in A$. Consequently, the environment moves into the next state $s_{t+1} \in S$, which is described by the transition function:

$s_{t+1}=f\left(s_{t}, a_{t}\right)$.

Moreover, the agent receives a feedback in form of a reward, which gets calculated by the reward function:

$r_{t+1}=J\left(s_{t}, a_{t}\right)$.

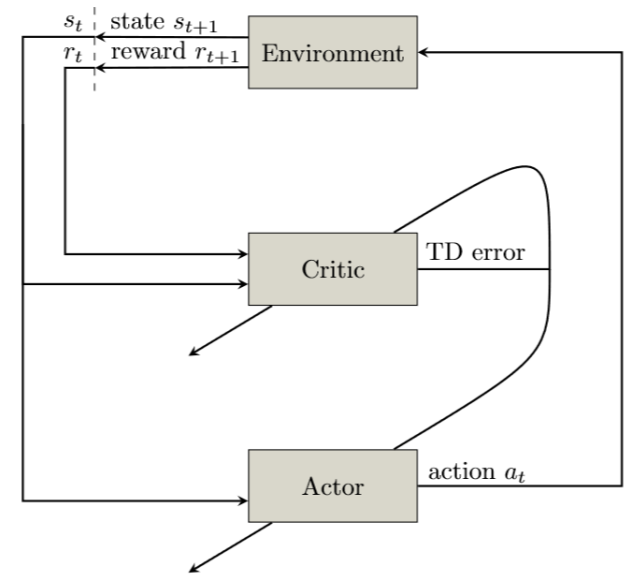

Figure 5: Visualization of the actor-critic structure. 
Summing up these rewards results in the discounted overall expected return:

$R_{t+1}=\sum_{t=0}^{\infty} \gamma^{t} r_{t+1}$,

with $R_{t+1} \in \mathbb{R}$ as the discounted return and $\gamma \in$ $[0,1]$ as a discount factor. This overall expected reward assumes that the agent performs an action in a certain state $s_{t}$ and then continues to follow the policy $\pi$. Throughout this paper, it is assumed that the policy is deterministic. To stay concise with already existing literature, $\mu$ refers to deterministic policies, while $\pi$ can represent both deterministic and stochastic policies.

In order to characterize such policies, stateaction value functions, namely Q-functions, are widely used by many RL algorithms. In a Qfunction, the overall expected reward for any state-action pair is stored. This correlation is defined as:

$Q\left(s_{t}, a_{t}\right)=\mathbb{E}\left[\sum_{t=0}^{\infty} \gamma^{t} r_{t+1}\right]$.

Since an RL agent is expected to pick the best action in the current state, the policy is given as follows:

$\mu\left(s_{t}\right)=\arg \max _{\mu} Q\left(s_{t}, a_{t}\right)$.

So, it is ensured that the policy returns always the action that maximizes the expected reward for a certain state.

Since the Q-function is usually unknown, the aim of RL is to iteratively calculate it. For this purpose, a wide range of approaches make use of the Bellman equation [7]:
$\mathrm{Q}\left(s_{t}, a_{t}\right)=\mathrm{r}\left(s_{t}, a_{t}\right)+\gamma \underset{a}{\max } Q\left(s_{t+1}, a_{t+1}\right)$.

This theory lays the foundation for all kinds of different RL algorithms, e.g. Q-learning [8], SARSA [9] or PoWeR [10].

Though, as it can be seen from the definitions the action and state spaces need to be finite and discrete. Since most real-world applications are continuous, those conditions are usually harmed and classic RL methods cannot be applied. This issue is often referred to as the "curse of dimensionality" [11]. To avoid this, function approximators are required to apply $\mathrm{RL}$ in continuous spaces.

\subsection{Deep deterministic policy gradient}

With the deep deterministic policy gradient (DDPG), Lillicrap et al. [4] introduced an algorithm that is able to solve continuous problems.

\section{Background}

Unlike standard RL algorithms, DDPG uses an actor-critic structure and neural networks as function approximators.

In the actor-critic setting, the agent is split into two models, namely the actor and the critic. The actor takes the state $s_{t}$ as input and computes an appropriate action $a_{t}$. The critic judges the action by calculating a Q-value from the input state $s_{t}$. This Q-value is compared to the actual reward $r_{t}$ and based on the resulting temporal difference

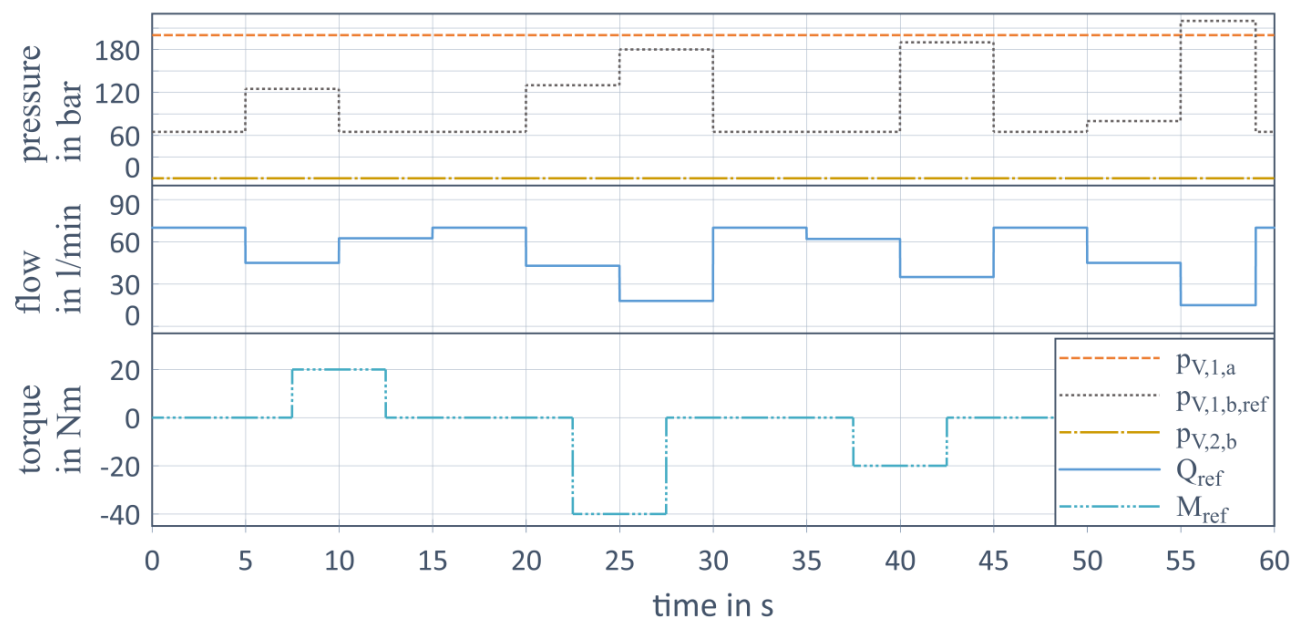

Figure 6: The test case used for training the agent. 
error, both actor and critic are optimized. So, it can be said that both parts of the agents are learning from each other's predictions.

In order to overcome the restriction of RL to discrete problems, both actor and critic are modeled as neural networks. Thus, continuous problems can be solved by the DDPG.

To ensure a more stable learning behavior, the algorithm uses soft target updates. This means that copies of both the critic and the actor network are created. These target networks are updated slower than the networks on which the learning is performed.

Additionally, a replay buffer is added to the algorithm in order to make it more data-efficient. This means that each tuple $\left(s_{t}, a_{t}, r_{t}, s_{t+1}\right)$ is saved for a given time. In each learning step, a batch with a predefined size is randomly sampled from the replay buffer. Hence, the algorithm learns not only from current observations, but also from experience.

\section{Algorithm}

To optimize the critic network, the loss $L \in \mathbb{R}$ needs to be minimized:

$L\left(\theta^{Q}\right)=\frac{1}{N} \sum_{j=1}^{N}\left(y_{j}-Q\left(s_{j}, a_{j} \mid \theta^{Q}\right)\right)^{2}$.

Here, $N \in \mathbb{N}$ is the number of samples that are taken from the replay buffer, $\theta^{Q} \in \mathbb{R}^{w}$ is the set of parameters that defines the critic network, and $w \in \mathbb{N}$ is the number of parameters of the critic

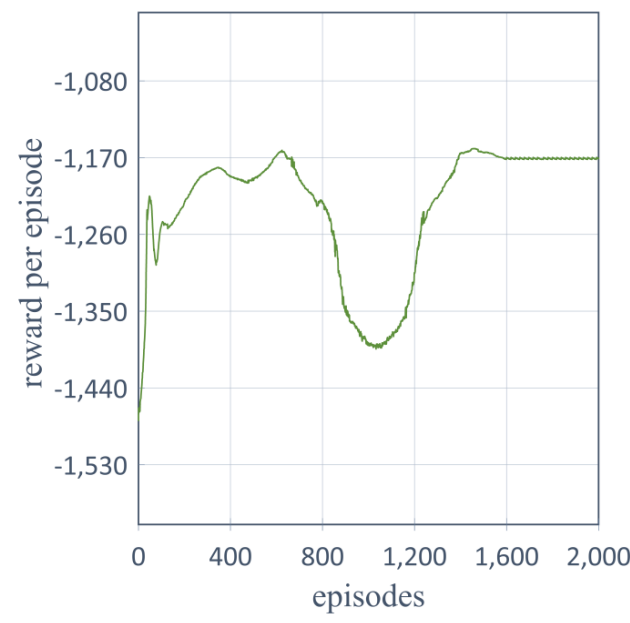

Figure 7: Visualization of the episodic reward.

network. Moreover, $y_{j} \in \mathbb{R}$ is the updated Qvalue, which is expressed by:

$y_{j}=r_{j}+\gamma Q^{\prime}\left(s_{j+1}, \mu^{\prime}\left(s_{j+1} \mid \theta^{\mu \prime}\right) \mid \theta^{Q^{\prime}}\right)$,

with $\mu^{\prime}$ as the policy that is defined by the target actor network, $\theta^{\mu \prime} \in \mathbb{R}^{o \prime}$ as the set of parameters that defines the target actor network, $o^{\prime} \in \mathbb{N}$ as the number of parameters of the target actor network, $Q^{\prime}$ as the Q-function that is formulated by the target critic network, $\theta^{Q^{\prime}} \in \mathbb{R}^{w \prime}$ as the set of parameters that defines the target critic network, and $w^{\prime} \in \mathbb{N}$ as the number of parameters of the target critic network. By comparing

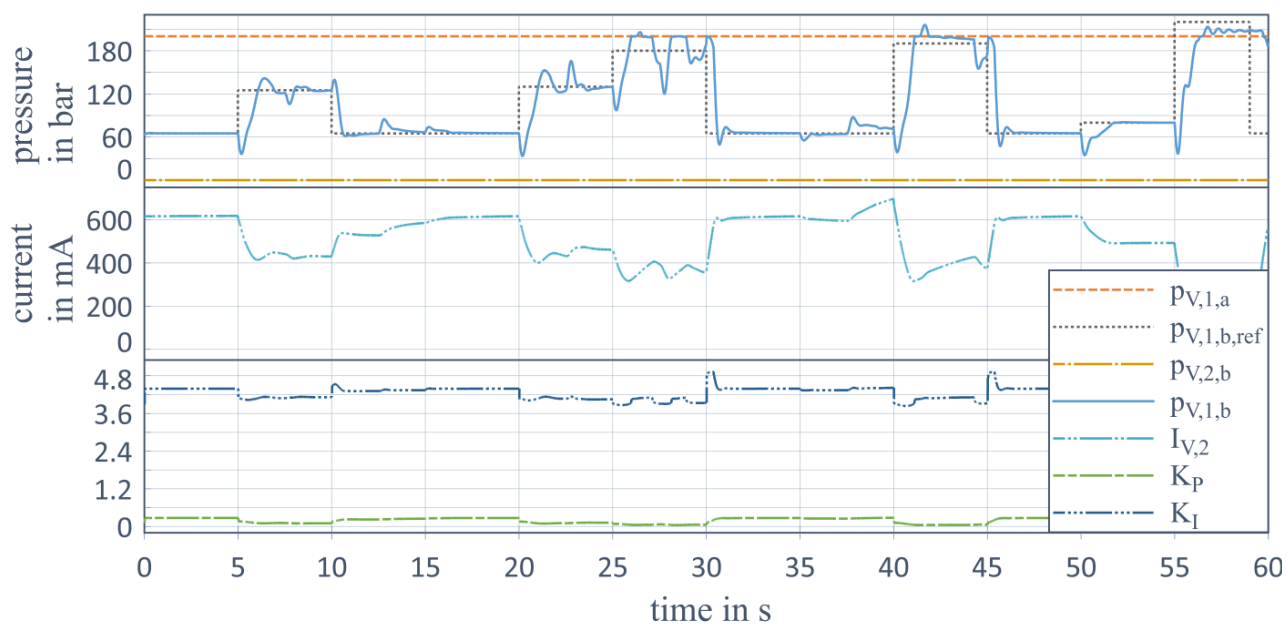

Figure 8: Performance of the learned agent in the simulative environment. 


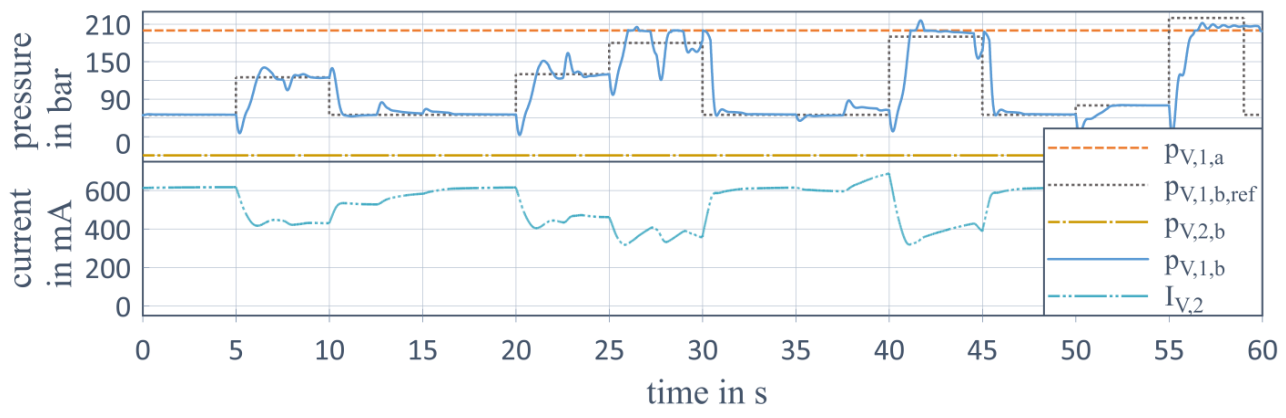

Figure 9: Performance of an expert tuned controller in the simulative environment.

Equation (22) with Equation (24), it can be seen that the update law is based on the Bellman equation.

The policy network is optimized by maximizing the expected return $J\left(\theta^{\mu}\right) \in \mathbb{R}$, which is defined as:

$J\left(\theta^{\mu}\right)=\mathbb{E}\left[\left.Q(s, a)\right|_{s=s_{t}, a=\mu\left(s_{t}\right)}\right]$.

Now, the policy gradient can be calculated via

$\nabla_{\theta^{\mu}} J\left(\theta^{\mu}\right) \approx$

$\left.\left.\nabla_{a} Q(s, a)\right|_{s=s_{t}, a=\mu\left(s_{t}\right)} \nabla_{\theta^{\mu}} \mu\left(s \mid \theta^{\mu}\right)\right|_{s=s_{t}}$.

Here, $\theta^{\mu} \in \mathbb{R}^{o}$ denotes the set of parameters that defines the actor network and $o \in \mathbb{N}$ the number of parameters of the actor network. Because the DDPG always takes a batch of samples for optimization from the replay buffer, the mean of the gradients in Equation (25) is used:

$$
\begin{aligned}
& \nabla_{\theta^{\mu}} J\left(\theta^{\mu}\right) \approx \\
& \frac{1}{N} \sum_{j=1}^{N}\left[\left.\left.\nabla_{a} Q(s, a)\right|_{s=s_{j}, a=\mu\left(s_{j}\right)} \nabla_{\theta^{\mu}} \mu\left(s \mid \theta^{\mu}\right)\right|_{s=s_{j}}\right] .
\end{aligned}
$$

Moreover, the target networks are updated as follows:

$\theta^{Q^{\prime}} \leftarrow \tau \theta^{Q}+(1-\tau) \theta^{Q^{\prime}}$,

$\theta^{\mu \prime} \leftarrow \tau \theta^{\mu}+(1-\tau) \theta^{\mu \prime}$,

with $\tau \in[0,1]$ as the update rate.

A block diagram of the actor-critic structure is displayed in Figure 5.

\section{EXPERIMENTAL SETTING}

This section explains, the experimental setting of system and agent.

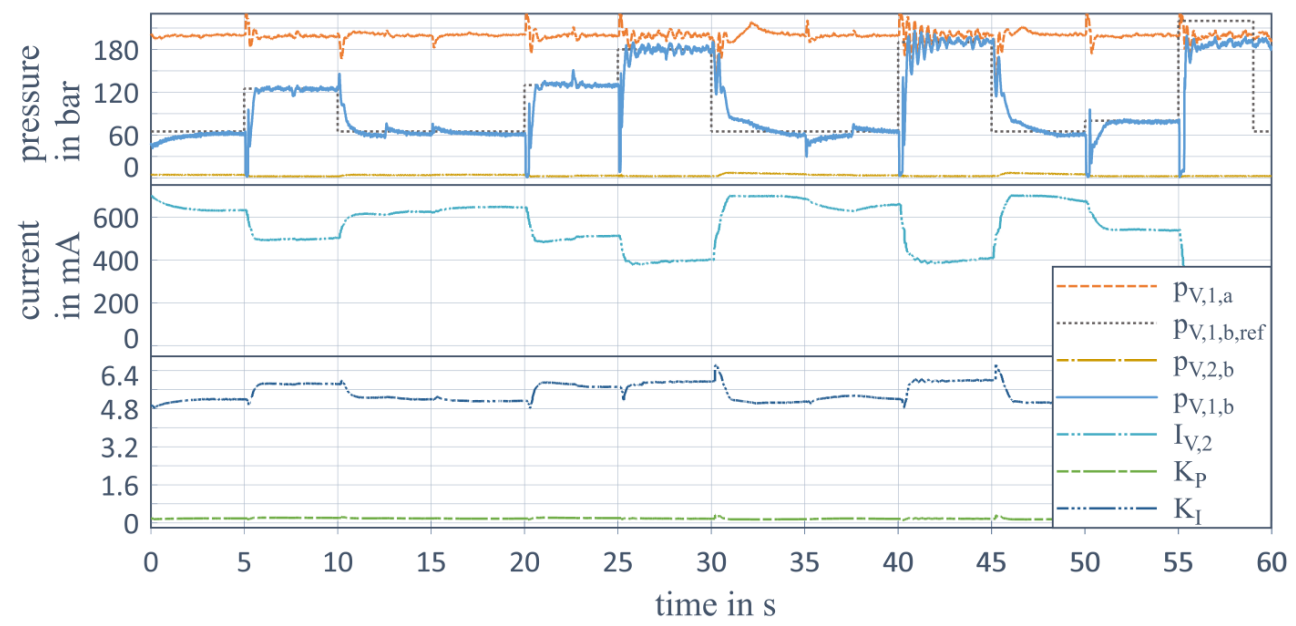

Figure 10: Performance of the learned agent on the test rig. 


\subsection{System setting}

In order to evaluate the proposed method, a simulative experiment is conducted. For this purpose, the algorithm is expected to track the pressure profile that can be seen in Figure 6. In the same figure, there can be seen a corresponding volumetric flow profile and a disturbance caused by the motor.

As previous experiments have shown, the proposed system reacts highly sensitive to the usage of the differential gain. Thus, $K_{d}$ remains 0 for all the experiments. This results in a PIcontroller, which means that the agent needs to set a proportional gain $K_{p}$ and an integral gain $K_{i}$.

While the system receives the values of those two gains from the agent, it is required to send back its resulting state.

The system's sample time is set to $5 \mathrm{~ms}$. Moreover, each 5th sample is evaluated by the reward function and stored into the replay buffer. Throughout the experiments, this setting has shown the best balance between the saving of computational power and accuracy.

In order to evaluate the performance of the system, the reward function is set as follows:

$r_{t}=-\exp \left(\frac{-1}{1+1.5 * 10^{-3} e^{2}+0.5 * 10^{-4}\left(\dot{p}_{V, 1, b}\right)^{2}}\right)$.

As it can be seen in Equation 30, the reward in each step is bounded in the interval $r_{t} \in$ $[-1,-\exp (-1)]$. Moreover, the reward depends on the control error $e$ and the pressure's rate of change $\dot{p}_{V, 1, b}$.

\subsection{Agent setting}

As explained in Chapter 3, the agent is designed in an actor-critic structure. The neural network (NN), which describes the actor, has 18 neurons in the input layer. Each of the following two hidden layers contains 256 neurons with rectified non-linearity [12] as activation. Moreover, there are 2 neurons in the sigmoidactivated output layer.

The critic NN consists of an input layer with 18 neurons. Both hidden layers consist of 256 neurons with rectified non-linearity as activation. The second hidden layer additionally includes the actions as input. The output layer consists of a neuron with linear activation.

In addition, it is to mention that the layers of all networks are fully connected. As input states, the agent receives the pressures
$p_{V, 1, a}, p_{V, 1, b}, p_{V, 2, a}, p_{V, 2, b}$ the reference pressure $p_{V, 1, b, r e f}$, the control error $e$, the currents $I_{V, 1}$ and $I_{V, 2}$, the reference volumetric flow $Q_{r e f}$, and the gradients of these values.

While the system state is sampled every $5 \mathrm{~ms}$, the agent is allowed to adapt the parameters every 50 samples, i.e. every $250 \mathrm{~ms}$. This restriction is based on the fact that the valve needs enough time to react to the changes. Faster changes would not be recognized by the hardware and, thus, not lead to any performance improvement. Thus, also policy updates are performed each $250 \mathrm{~ms}$ during the learning phase.

The complete parameter settings for the training of the agent are listed in Table 1.

Table 1: Parameters of the DDPG agent

\begin{tabular}{ll}
\hline DDPG parameter & Value \\
\hline Actor learning rate & 0.000015 \\
Critic learning rate & 0.00015 \\
Replay buffer size & 100000 \\
Batch size & 32 \\
$\gamma$ & 0.0 \\
$\tau$ & 0.001 \\
$K_{P}$ bound & {$[0,10]$} \\
$K_{I}$ bound & {$[0,20]$} \\
\hline
\end{tabular}

\section{EXPERIMENTAL EVALUATION}

The upcoming section starts with an evaluation of the simulative experiments. Afterwards, results from hardware tests are presented.

\subsection{Simulative experiment}

The simulative experiment is set up as explained in the previous section and starts with the training of the DDPG agent. The test case from Figure 6 is simulated for 2000 epochs. While a single epoch equals 60 seconds (real-time) of interaction, the agent gets 2000 minutes of interaction time with the system the learn an appropriate control law.

As Figure 7 shows, the learning process converges to a reward of about -1170 after about 1600 epochs. So, it can be assumed that the agent is able to find an optimal solution for the control problem.

In order to evaluate the learned result, the agent is evaluated on the test case and compared to an expert-tuned controller. Figure 8 shows the 
performance of the agent's policy. By comparing this result with that of the expert-tuned controller, which can be seen in Figure 9, the behavior is nearly identical. This is also supported by the overall achieved rewards, which is -1165.02 for the agent and -1166.84 for the expert.

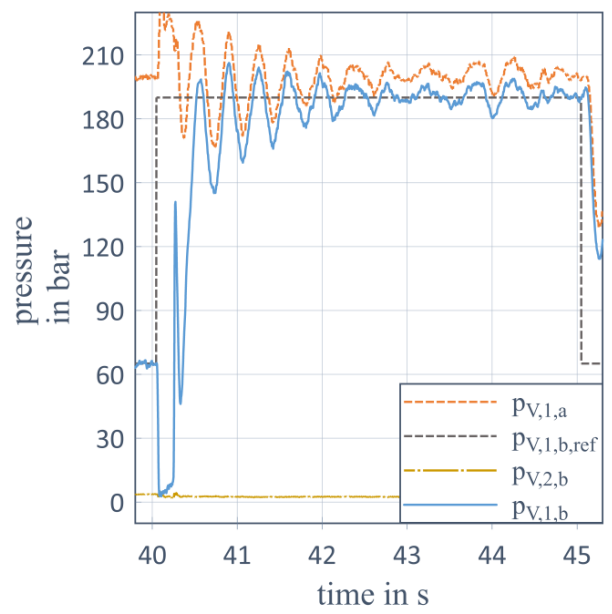

Figure 11: Visualization of a descending oscillation.

\subsection{Hardware experiment}

Since the method shows promising results in the simulation environment, it is also evaluated on real hardware. The same system as described in Chapter 2 is build up as a test-rig.

In the hardware setting, a training phase cannot be conducted, because that would mean to run the test rig nonstop for more than 30 hours. Moreover, the hardware could be damaged, if the agent tries to set PI-values that destabilize the system. To overcome these issues, the learned agent from the simulative experiment is directly applied to the test rig.

The result of the experiment can be seen in

Figure 10. Here, the agent accurately follows the desired pressure profile $p_{V, 1, b, r e f}$. By comparing this with the result from Figure 8, it can be even said that the overall performance is comparable to that in the simulative environment.

This is remarkable as there are several differences between the simulation and the test rig. The major one might be the pressure $p_{V, 1, a}$. In the simulation, this value is assumed as constant, since there is no implementation of the pump model. Though, in the test rig $p_{V, 1, a}$ might change or even oscillate in some situations. As a result, also $p_{V, 1, b}$ starts to oscillate, e.g. between $40 \mathrm{~s}$ and $45 \mathrm{~s}$ (see Figure 11). However, the oscillation decays over time, which is acceptable in this test scenario.

Moreover, also $p_{V, 2, b}$ is not as ideal as in simulation, where this value is simply set to 0 bar. Though, also this causes no trouble for the agent.

In addition, the motor torque $M$ is much smoother in the test rig than in the simulation. This comes from the fact that the real motor cannot perform jumps as the motor model from the simulation. So, the disturbance caused by the motor is applied smoother, but for a longer time interval.

\section{CONCLUSION AND OUTLOOK}

This paper shows that PID-controllers for hydraulic systems can be autonomously learned by RL algorithms. The used DDPG algorithm achieves a performance that is comparable to an expert-tuned controller within an acceptable simulation time.

Additionally, the evaluation on a test rig shows that a simplified system model is sufficient to transfer knowledge gained in simulation to a real hardware application without a considerable drop in performance. The algorithm is even robust against previously unknown system behavior and disturbances. This implies that the agent is somehow able to generalize its experience.

For future work, it will be necessary to further explore this generalization behavior, e.g. to a wider range of test cases or even different hardware components inside the test rig. Moreover, a strategy for fine-tuning or learning on the hardware is required to enable RL to a wider range of applications in hydraulic control systems. In this context, a thorough stability analysis of the method and the learned controller might be required.

Finally, it can be said that reinforcement learning methods have a great potential for making hydraulic control systems more efficient and flexible. 


\section{NOMENCLATURE}

Throughout the work, it can be assumed that derivations and gradients are performed with respect to time. Any exceptions are explicitly specified.

\begin{tabular}{|c|c|}
\hline$D D P G$ & Deep Deterministic Policy Gradients \\
\hline$R L$ & Reinforcement Learning \\
\hline$M D P$ & Markov Decision Process \\
\hline$N N$ & Neural Network \\
\hline$\varphi$ & Rotation angle in rad \\
\hline$\dot{\varphi}$ & Angular velocity in $\mathrm{rad} / \mathrm{s}$ \\
\hline$\ddot{\varphi}$ & Angular acceleration in $\mathrm{rad} / \mathrm{s}^{2}$ \\
\hline$I_{V, i}$ & Control current in $\mathrm{mA}$ \\
\hline$M$ & Torque in $\mathrm{Nm}$ \\
\hline$c_{i}$ & Bulk modulus and fluid volume in $\mathrm{kg} /\left(\mathrm{m}^{4} \mathrm{~s}^{2}\right)$ \\
\hline$d$ & Damping factor in $\mathrm{Nm} \mathrm{s} / \mathrm{rad}$ \\
\hline$V_{d}$ & Reference volume in 1 \\
\hline$I$ & Moment of inertia in $\mathrm{kg} \mathrm{m}^{2}$ \\
\hline$Q$ & Volumetric flow in $1 / \mathrm{min}$ \\
\hline$A_{i}()$. & Valve opening in $\mathrm{mm}^{2}$ \\
\hline$\rho$ & Fluid density in $\mathrm{kg} / \mathrm{m}^{3}$ \\
\hline$i$ & Index denoting valve number \\
\hline$j$ & Counter variable \\
\hline$\alpha$ & Discharge coefficient \\
\hline$t$ & Time in $\mathrm{s}$ \\
\hline$x$ & State vector of the dynamic system \\
\hline$\dot{x}$ & Derivative of the state vector \\
\hline$S, s_{t}$ & Set of states and state at time $t$ \\
\hline$A, a_{t}$ & Set of actions and action at time $t$ \\
\hline$u$ & PID control output in $\mathrm{mA}$ \\
\hline$K_{P}$ & Proportional control gain \\
\hline$K_{I}$ & Integral control gain \\
\hline$K_{D}$ & Derivative control gain \\
\hline$e$ & Control error in bar \\
\hline$R, r_{t}$ & Overall expected reward and reward at time $t$ \\
\hline$J$ & Reward function \\
\hline$\gamma$ & Discount factor \\
\hline$Q()$. & Q-function \\
\hline$\mu()$. & Policy function \\
\hline$L()$. & Loss function \\
\hline$\theta()$. & Critic function \\
\hline$p_{V, i, a}$ & Pressure on valve's a-side in bar \\
\hline$p_{V, i, b}$ & Pressure on valve's b-side in bar \\
\hline$\theta^{\mu}, \theta^{\mu \prime}$ & Set of parameters of target network \\
\hline$\theta^{Q}, \theta^{Q^{\prime}}$ & Set of parameters of actor network \\
\hline$o, o^{\prime}$ & Number of parameters in actor network \\
\hline$w, w^{\prime}$ & Number of parameters in critic network \\
\hline$y_{j}$ & Updated Q-value \\
\hline$\tau$ & Update rate \\
\hline
\end{tabular}

\section{REFERENCES}

[1] Bischoff B, Nguyen-Tuong D, Koller T, Markert H, Knoll A (2013) Learning Throttle Valve Control Using Policy Search. In: Blockeel H, Kersting K, Nijssen S, Železný F (eds) Machine Learning and Knowledge Discovery in Databases. ECML PKDD 2013. Lecture Notes in Computer Science, vol 8188. Springer, Berlin, Heidelberg

[2] Kim J, Jeon J, Heo H (2011) Design of adaptive PID for pitch control of large wind turbine generator. 10th International Conference on Environment and Electrical Engineering, Rome, Italy

[3] Deisenroth M, Rasmussen CE (2011) PILCO: A model-based and data-efficient approach to policy search. In: Lise G, Scheffer $\mathrm{T}$ (eds) Proceedings of the 28th International Conference on Machine Learning, Bellevue, July 2011, Omnipress Publishing, Madison

[4] Lillicrap T, Hunt J, Pritzel A, Heess N, Erez T, Tassa Y, Silver D, Wierstra D (2016) 4th International Conference on Learning Representations. San Juan, Puerto Rico, May 24, 2016

[5] Akkaya AV (2006) Effect of Bulk Modulus on Performance of a Hydrostatic Transmission Control system. Sadhana 543 - 556

[6] Sutton RS, Barto AG (1998) Reinforcement Learning - An Introduction. MIT Press, Cambridge, USA

[7] Bellman RE (1957) Dynamic Programming. Princeton University Press, Princeton, USA

[8] Rummery GA, Niranjan M (1994) On-Line QLearning Using Connectionist Systems. University of Cambridge, Department of Engineering Cambridge, England

[9] Watkins CJCH (1989), Learning from Delayed Rewards. King's College, Cambridge, England.

[10] Kober J, Peters JR (2008) Policy Search for Motor Primitives in Robotics. In: Advances in Neural Information Processing Systems 21. 22nd Annual Conference on Neural Information Processing Systems, British Columbia, Canada, December 8-11, 2008, p 849

[11] Bellman RE (1961) Adaptive Control Processes. Princeton University Press, Princeton USA

[12] Glorot X, Bordes A, Bengio Y (2011) Deep sparse rectifier neural networks. In. Proceedings of the Fourteenth International Conference on Artificial Intelligence and Statistics, Fort Lauderdale, USA, April 11-13, 2011, p 315 